\title{
Critérios para inserção de estratégias cognitivas e metacognitivas no desenvolvimento de lógica de programação em ambientes virtuais de aprendizagem
}

\author{
Carla Lopes Rodriguez ${ }^{1,}$ Rafaela Vilela da Rocha ${ }^{1}$, Denise Hideko Goya ${ }^{1}$, \\ Mirtha Lina Fernandéz Venero ${ }^{1}$, Francisco Zampirolli ${ }^{1}$ \\ ${ }^{1}$ Centro de Matemática, Computação e Cognição - Universidade Federal do ABC \\ CEP: 09210-580 - Santo André - SP - Brasil \\ \{c.rodriguez, rafaela.rocha, denise.goya, mirtha.lina, \\ fzampirolli\}@ufabc.edu.br
}

Abstract. Several studies have indicated that mobilizing cognitive and metacognitive strategies can improve student performance. This article describes criteria that support the use of these strategies to develop programming logic, and presents a checklist that relates them with resources and tools that can help the teacher to promote their uses in virtual learning environments. Criteria, resources and tools have been specified to support the use of these strategies. A learning situation was conducted, and later evaluated by students and teachers, corroborating the validation of these strategies and criteria and the necessity of their use in computational environments.

Resumo. Vários estudos têm indicado que mobilizar estratégias cognitivas e metacognitivas pode melhorar o desempenho dos alunos. Este artigo descreve critérios que apoiam o uso dessas estratégias no desenvolvimento da lógica de programação e apresenta um checklist que as relaciona com recursos $e$ ferramentas que podem auxiliar o professor a promover seus usos em ambientes virtuais de aprendizagem. Critérios, recursos e ferramentas foram especificados para apoiar o uso dessas estratégias. Uma situação de aprendizagem foi conduzida e, posteriormente, avaliada corroborando para a validação da viabilidade da aplicação desses critérios e a necessidade de implementação das estratégias em ambientes computacionais.

\section{Introdução}

O sucesso no desempenho escolar está fortemente atrelado ao uso de diversas estratégias de aprendizagem, que atuam sobre as estruturas cognitivas [Flavell 1976, Silva e Sá 1997, Boruchovitch 1999, Almeida 2002]. Essas estratégias são procedimentos para armazenar, recuperar e processar informações de forma eficiente (cognitivas), assim como planejar, monitorar, regular e avaliar o processo cognitivo (metacognitivas). Entre as estratégias cognitivas estão, por exemplo, as de ensaio ou revisão (repetição verbal ou escrita), organização e elaboração do conteúdo (de resumos, perguntas e respostas, exercícios, gráficos e mapas mentais). As estratégias metacognitivas envolvem os processos de tomada de consciência e reflexão sobre o próprio sistema cognitivo. Weinstein e Mayer (1985) também reconhecem as estratégias afetivas, relacionadas ao controle da ansiedade, as mudanças emocionais e frustração em situações de avaliação, como importantes para o desempenho dos estudantes. 
VII Congresso Brasileiro de Informática na Educação (CBIE 2018)

Anais do XXIX Simpósio Brasileiro de Informática na Educação (SBIE 2018)

Na Educação a Distância (EAD), a mobilização de diversas estratégias de aprendizagem é fundamental, pois os estudantes precisam de maior autonomia [Chen 2002, Testa e Freitas 2006, Fantinel et al. 2013, Castro 2016]. No entanto, existem poucos trabalhos que detalham como professores e estudantes podem mobilizar essas estratégias em cursos de lógica de programação semipresenciais ou EAD [Pimentel et al. 2005]. Nesse contexto, uma iniciativa desenvolvida para a disciplina Processamento da Informação (PI) semipresencial, do curso Bacharelado em Ciência \& Tecnologia (BC\&T), da Universidade Federal do ABC (UFABC), buscou identificar quais estratégias os alunos mobilizam para aprender o conteúdo da disciplina. PIsemipresencial é ofertada por meio de um Ambiente Virtual de Aprendizagem (AVA). O material com o conteúdo da disciplina está disponível no AVA e é composto de slides e videoaulas que devem ser acompanhadas pelos alunos semanalmente. Para cada aula são propostas atividades (enunciados de problemas para elaboração de códigos) e exercícios teóricos (testes de múltipla escolha). A interação entre alunos, professores e monitores é viabilizada por meio das ferramentas de comunicação do AVA, no caso, a ferramenta mensagens e a caixa de texto para devolutiva das atividades. Apenas a aula inaugural e as três avaliações são realizadas presencialmente.

Ofertada desde 2013, a taxa de evasão e retenção na disciplina PI sempre foi alta e continuava a crescer a cada ano, em parte por conta do perfil heterogêneo dos alunos, pois o curso é interdisciplinar. Os resultados encontrados nas respostas dos alunos ao formulário de avaliação final de PI-semipresencial indicava algumas fragilidades nos materiais disponíveis para o estudo do conteúdo proposto. Assim, em 2017, foi proposto um projeto ${ }^{1}$ que teve, inicialmente, o objetivo de desenvolver materiais complementares para apoio à aprendizagem do conteúdo de PI [Goya et al. 2017 e Rodriguez et al. 2017]. Contando com três monitores bolsistas, foram desenvolvidos resumos, no formato de slides, que sintetizavam e destacavam os conceitos teóricos mais importantes trabalhados na disciplina, além de exemplos de aplicação prática desses conceitos. Entretanto, observou-se que poucos alunos acessavam os materiais extra disponíveis, e o percentual de alunos reprovados continuou alto, principalmente nos quadrimestres considerados não "ideais", isto é, fora da grade recomendada ${ }^{2}$. Paralelamente à disponibilização dos materiais extras passou-se a investigar quais as estratégias cognitivas e metacognitivas os alunos de PI-semipresencial mobilizavam durante o percurso de aprendizagem.

Diante do que foi exposto, este artigo descreve um conjunto de critérios que visam apoiar o uso de estratégias de aprendizagem cognitivas e metacognitivas para o desenvolvimento de lógica de programação, pelos alunos, bem como apoiar o planejamento e inserção dessas estratégias em AVAs, pelos professores. Na Seção 2, é apresentada a fundamentação teórica e os trabalhos relacionados. Na Seção 3, estão alguns resultados alcançados em uma situação de aprendizagem conduzida no âmbito do projeto Met@Aprendiz@gem. Na Seção 4, são descritos os critérios que derivam das estratégias de aprendizagem cognitivas e metacognitivas, e respectivo checklist para sua implementação. Por fim, as discussões (Seção 5) e considerações finais (Seção 6).

\footnotetext{
${ }^{1}$ O projeto "Recursos e estratégias metacognitivas para apoiar a construção do pensamento lógico" teve como objetivos propor "trilhas de aprendizagem" diferenciadas para um mesmo conteúdo.

2 Os estudantes que se matriculam em PI em quadrimestres não ideais ( $2^{\circ}$ ou $3^{\circ}$ de cada ano), quase sempre se encontram em uma das seguintes situações: ou estão refazendo a disciplina, ou não seguem a grade recomendada por terem apresentado dificuldades em outras disciplinas.
} 
VII Congresso Brasileiro de Informática na Educação (CBIE 2018)

Anais do XXIX Simpósio Brasileiro de Informática na Educação (SBIE 2018)

\section{Referencial Teórico e Trabalhos Relacionados}

De acordo com Flavell (1976), a metacognição implica o conhecimento sobre o próprio conhecimento (metaconhecimento ou conhecimento metacognitivo), assim como sobre a natureza das tarefas cognitivas, as estratégias para sua solução e competências executivas para monitorar as atividades cognitivas. Diversas habilidades estão associadas ao processo metacognitivo: planejamento, controle executivo, reflexão, autorregulação, monitoramento, autoavaliação [Brown 1987, Weinert \& Kluwe 1987, Son e Schwartz 2002]. Por isso, conforme Leite e Darsie (2011), as atividades metacognitivas permitem um desenvolvimento superior da capacidade de "aprender a aprender". Dessa forma, o estudante adquire autonomia intelectual e controla, monitora e regula suas experiências cognitivas.

As estratégias de aprendizagem podem ser desenvolvidas de forma intuitiva e natural ou treinadas para aumentar a efetividade da aprendizagem [Warr e Allan, 1998]. No entanto, segundo Beyer (1985), o desenvolvimento da metacognição não acontece naturalmente; por isso o professor deve propor um repertório de estratégias e realizar atividades que promovam o seu uso e desenvolvimento [Moreira 2014]. Esse desenvolvimento evolui com o avanço da idade e as experiências cognitivas, fazendo o estudante escolher a melhor estratégia de acordo com o seu objetivo de aprendizagem. Exemplos de como mobilizar estratégias metacognitivas são: sugerir que os alunos pensem em voz alta; auxiliá-los a descobrir como superar as dificuldades com determinado conteúdo; estimular perguntas antes, durante e depois da realização de uma tarefa; ajudar a perceber conexões, relações, similaridades e diferenças; perguntar pelos resultados e o procedimento para obtê-los; torná-los conscientes dos critérios de avaliação [Leite e Darsie 2011 apud Koutelini 1991 p. 52-53].

Os trabalhos de Alhazbi et al. (2010), Alaoutinen e Smolander (2010) e Parham et al. (2010) reforçam o fato dos diversos aspectos metacognitivos terem papel significativo não somente para o raciocínio lógico como também na resolução computacional de problemas. Outras pesquisas mostram que o uso de estratégias metacognitivas (e.g. autorregulação) geram melhor desempenho acadêmico em cursos introdutórios de Programação [Bergin et al. 2005, Pimentel et al. 2005, Havenga et al. 2013, Lishinski et al. 2016], e no desenvolvimento de programas orientados a objetos [Havenga 2015]. No entanto, poucos trabalhos abordam a inserção da metacognição em AVAs e sistemas tutores, sendo a maior parte com foco na autoavaliação e automonitoramento [Brusilovsky e Sosnovsky 2005, Pimentel et al. 2005, Ngai et al. 2009, Kautzmann e Jaques 2016]. Algumas iniciativas abordam o uso de feedback para estimular a autoavaliação [Krebs 2005, Moreira e Favero 2009, Sirotheau et al. 2011], enquanto outras a avaliação por pares [Sitthiworachart e Joy 2004]. Francisco et al. (2016) apresentaram uma revisão sistemática de juízes online para o ensino de programação elencando um conjunto de requisitos funcionais e não funcionais que devem ser atendidos. Os autores apontaram a importância de definir padrões que permitam a comparação formal e rigorosa das ferramentas usadas no ensino de programação. As estratégias de aprendizagem ${ }^{3}$ que os alunos mobilizam para apreender o conteúdo de lógica de programação foram exploradas por Goya et al. (2017). Foi aplicado um questionário de autoavaliação, logo após a primeira prova, com questões sobre as estratégias cognitivas e metacognitivas mobilizadas pelos alunos. O foco da

\footnotetext{
${ }^{3}$ Estratégias de aprendizagem adaptadas de Santos e Boruchovitch (2008). https://goo.gl/aW5tmY
} 
VII Congresso Brasileiro de Informática na Educação (CBIE 2018)

Anais do XXIX Simpósio Brasileiro de Informática na Educação (SBIE 2018)

pesquisa estava em analisar as estratégias e sua influência no desempenho dos alunos ao longo da disciplina. O questionário foi respondido por 133 alunos e revelou, principalmente, que os participantes da pesquisa adotavam com mais frequência estratégias de aprendizagem metacognitivas do que estratégias cognitivas. Dentre as estratégias metacognitivas menos usadas, destacaram-se as seguintes: i) Reunir com os colegas para estudar; ii) Colar lembretes para recordar o que é preciso fazer; iii) Pedir auxílio ao professor/monitor sobre dúvidas na matéria; iv) Anotar em uma agenda o que há para fazer; v) Pedir ajuda aos colegas em caso de dúvidas; vi) Controlar a ansiedade em situações de avaliação; vii) Rever as anotações feitas durante os momentos de estudo. Em relação às estratégias de aprendizagem cognitivas, as menos mobilizadas são as que requerem que aluno tome a iniciativa de refletir sobre o assunto estudado. Por exemplo, elaborar perguntas e respostas sobre o assunto, identificar as ideias principais e relacioná-las através de diagramas ou mapas conceituais mais complexos.

\section{Projeto Met@Aprendiz@gem}

O projeto “Met@Aprendiz@gem: recursos e estratégias de ensino e aprendizagem para apoiar a construção do pensamento lógico", visa propor iniciativas pedagógicas capazes de mobilizar as estratégias cognitivas e metacognitivas pouco usadas pelos alunos matriculados na disciplina de PI além de desenvolver recursos e materiais para apoiar o ensino [Goya et al. 2017; Rodriguez et al. 2017].

Em 2018, o projeto passou a contar com a participação de alunos monitores (dez bolsistas e dois voluntários). Foram definidas quatro frentes de trabalho: i) desenvolvimento de materiais, que envolve a pesquisa de ferramentas e materiais e o desenvolvimento e aplicação desses recursos em situações concretas de aprendizagem; ii) plantões de monitoria presencial e virtual. Os plantões presenciais são realizados pelos alunos monitores em dias/horários fixos, e em laboratório específico. O plantão virtual é realizado via página do projeto no Facebook $^{4}$; iii) desenvolvimento e manutenção do site do projeto ${ }^{5}$, que é um espaço digital de divulgação e de realização de ações de aprendizagem; e iv) pesquisa, que tem como ação principal investigar outras iniciativas sobre o uso de estratégias para o ensino-aprendizagem de lógica de programação, e apoiar a análise dos dados coletados durante o projeto.

Dado que o primeiro quadrimestre (2018.Q1) é o quadrimestre "ideal" para os alunos, foi proposta uma ação de aprendizagem, antes da realização da primeira prova (P1), denominada de "Desafio PI". A ação tinha como objetivo desafiar os alunos com problemas de dificuldades variadas, permitir a discussão de soluções em equipes e ser uma revisão do conteúdo (estrutura sequencial, seleção e laços) para que os alunos de ambas as modalidades (presencial e semipresencial) pudessem se preparar para a P1.

Considerando as estratégias de aprendizagem, identificadas anteriormente como as menos mobilizadas pelos alunos de PI, foi proposta uma ação presencial, baseada no modelo de França e Tedesco (2014), com duração de quatro horas, subdividida em três momentos distintos e complementares:

- Momento 1: participação individual, com duração de aproximadamente 50 minutos.

Preenchimento de um formulário de autoavaliação sobre o grau de confiança nos

\footnotetext{
${ }^{4}$ Página do projeto Met@ Aprendiz@gem no FB: www.facebook.com/metaprendizagem/

5 Site do Met@Aprendiz@gem: http://pesquisa.ufabc.edu.br/lirte/metaprendizagem/
} 
VII Congresso Brasileiro de Informática na Educação (CBIE 2018)

Anais do XXIX Simpósio Brasileiro de Informática na Educação (SBIE 2018)

conhecimentos prévios, relacionados aos conteúdos anteriormente estudados. A estratégia mobilizada estava relacionada principalmente à reflexão (autorregulação) sobre o próprio percurso de aprendizagem [Pimentel et al. 2005]. Posteriormente, cada aluno recebeu três enunciados de problemas com níveis diferenciados, do mais complexo ao mais simples, sendo que a solução do problema de menor dificuldade estava embutida na solução do mais complexo; o aluno deveria escolher um e resolvê-lo individualmente em 40 minutos.

- Momento 2: participação em grupos pequenos, com duração de aproximadamente 30 minutos. A estratégia de aprendizagem mobilizada envolveu estimular a colaboração entre os alunos. Os grupos foram orientados a discutir as soluções encontradas individualmente, e a identificar os erros cometidos. $\mathrm{O}$ momento foi introduzido por uma breve explanação sobre a importância do uso de estratégias metacognitivas para o aprendizado e recomendações para que os alunos se atentassem àquelas menos mobilizadas.

- Momento 3: participação no grupo geral. Para encerrar o Desafio PI foi realizada uma revisão do conteúdo estudado, e apresentada a solução passo a passo do exercício mais complexo. A estratégia de aprendizagem mobilizada nesse momento envolveu favorecer a reflexão sobre os exercícios praticados e auxiliar os alunos a sanar dúvidas que ainda pudessem persistir.

Como resultado do Desafio PI esperava-se que os alunos seguissem se preparando para a $\mathrm{P} 1$, e que se sentissem mais confortáveis e seguros em relação à situação de prova que viriam a enfrentar. Se inscreveram no Desafio 119 alunos. Outros 43 alunos manifestaram interesse, mas ficaram em lista de espera, dada a restrição de espaço na sala de aula. No dia do encontro, estiveram presentes 68 alunos. A identificação nas atividades entregues foi opcional, para favorecer maior credibilidade à autoavaliação. Ainda assim, 60 alunos se identificaram e essa quantidade foi usada para mapear os resultados da ação. Dentre os 38 alunos de PI-semipresencial inscritos, apenas 19 compareceram e se identificaram (11,7\% da turma). Dentre os 81 alunos de PI presencial inscritos, estiveram presentes apenas 41 (4,0\% dos alunos das turmas).

Após a realização da P1 um questionário de autoavaliação foi respondido pelos alunos. Além de questões específicas sobre o uso de estratégias de aprendizagem, foram incluídas questões sobre o Desafio PI. Ao analisar os dados da turma de PIsemipresencial, detectou-se que 121 alunos responderam a autoavaliação $(74,7 \%$ da turma); os que participaram do Desafio e responderam a autoavaliação somaram 19 alunos (11,7\%). Embora essa quantidade seja relativamente pequena, foi possível extrair algumas impressões sobre a aceitação da proposta. Dentre esses participantes, 12 alunos $(63,2 \%)$ afirmaram que o encontro foi útil e os ajudou a se preparar melhor para a P1; $21,1 \%$ gostaria de participar de futuros encontros; e 31,6\% afirmaram que pouco ajudou. Por meio de comentários fornecidos, foi possível identificar que entre os aspectos que frustraram a expectativa dos alunos houve a percepção (equivocada) de que os desafios foram mais simples do que a prova real, ou de que o encontro deveria ter sido apenas um simulado de prova; alguns julgaram curto o tempo para resolver os exercícios ou queriam mais tempo para revisão ou para explicação das soluções. Ainda sobre os dados de PI-semipresencial, dentre os que não participaram do encontro, 46 alunos $(28,4 \%$ da turma) afirmaram que o motivo foi falta de tempo e $7,4 \%$ não conseguiram vaga; 59 alunos (36,4\% da turma) afirmaram que gostariam de participar de outros encontros. Os dados sugerem que alguns alunos da modalidade semipresencial podem ter se beneficiado com a ação e que outros poderiam vir a se beneficiar. 
VII Congresso Brasileiro de Informática na Educação (CBIE 2018)

Anais do XXIX Simpósio Brasileiro de Informática na Educação (SBIE 2018)

\section{Critérios para inserção de estratégias cognitivas e metacognitivas no desenvolvimento de lógica de programação}

A partir de uma revisão de literatura, e dos resultados do Desafio PI, ação realizada no âmbito do projeto Met@ Aprendiz@gem, foram selecionados conceitos, teorias, recursos e ferramentas que embasam a escolha de determinadas estratégias cognitivas e metacognitivas para o ensino e a aprendizagem de lógica de programação [Goya et al. 2017], sendo especificados 11 critérios subdivididos em: estratégias cognitivas (cinco) e metacognitivas (seis).

Considerando a importância de inserir as estratégias de aprendizagem no processo de ensino, o Quadro 1 apresenta os critérios para as estratégias cognitivas, que podem apoiar a aprendizagem de lógica de programação. Para cada critério definido é apresentado um conjunto de recursos e ferramentas que podem auxiliar o professor a promover a inserção do critério em sua prática docente (Quadro 1, segunda coluna). Para apoiar o aluno em seu percurso de aprendizagem, o Quadro 1 (terceira coluna) apresenta um conjunto de recursos e ferramentas que podem auxiliar o estudante a promover a inserção do critério selecionado em sua rotina de estudos.

Quadro 1. Critérios e checklist de estratégias cognitivas

\begin{tabular}{|l|l|l|}
\hline $\begin{array}{c}\text { Critérios para estratégias } \\
\text { cognitivas }\end{array}$ & $\begin{array}{l}\text { Checklist com recursos e ferramentas para } \\
\text { o professor promover o critério }\end{array}$ & Checklist de recursos e ferramentas para os alunos \\
\hline $\begin{array}{l}\text { 1. Anotar explicações do } \\
\text { professor }\end{array}$ & Ferramenta de anotações & $\begin{array}{l}\text { Tenha sempre um caderno ou ferramenta para anotação, } \\
\text { enquanto estiver consultando o material instrucional }\end{array}$ \\
\hline $\begin{array}{l}\text { 2. Fazer resumos, esquemas, } \\
\text { diagramas }\end{array}$ & $\begin{array}{l}\text { Atividades usando ferramentas externas } \\
\text { plugins e outras, como mapa mental, p.ex) }\end{array}$ & $\begin{array}{l}\text { Elenque os conceitos vistos, descrevendo o que é e para } \\
\text { que serve cada um }\end{array}$ \\
\hline $\begin{array}{l}\text { 3. Elaborar perguntas e } \\
\text { respostas }\end{array}$ & FAQ (questões mais frequentes) & $\begin{array}{l}\text { No material instrucional, identifique se cada novo } \\
\text { conceito está associado a um novo problema ou se ele } \\
\text { otimiza algo previamente conhecido; } \\
\text { Nos exemplos, identifique se poderiam ser feitos de } \\
\text { forma diferente; se sim, compare }\end{array}$ \\
\hline 4. Pesquisar materiais extra & Fóruns para alunos proporem e debaterem & $\begin{array}{l}\text { Consulte a relação de materiais complementares ou } \\
\text { pesquise materiais adicionais }\end{array}$ \\
\hline $\begin{array}{l}\text { 5. Fixar conceitos e padrões } \\
\text { de soluções de problemas }\end{array}$ & Ferramenta de quiz & $\begin{array}{l}\text { Relacione palavras reservadas, sintaxes, funções de } \\
\text { biblioteca importantes }\end{array}$ \\
\hline
\end{tabular}

Da mesma forma, o Quadro 2, apresenta os critérios para estratégias metacognitivas, que podem apoiar o ensino (coluna 2) e a aprendizagem de lógica de programação (coluna 3).

\section{Quadro 2. Critérios e checklist de estratégias metacognitivas}

\begin{tabular}{|l|l|l|}
\hline \multicolumn{1}{|c|}{$\begin{array}{c}\text { Critérios para } \\
\text { estratégias } \\
\text { metacognitivas }\end{array}$} & $\begin{array}{l}\text { Checklist com recursos e ferramentas para } \\
\text { o professor promover o critério }\end{array}$ & \multicolumn{1}{c|}{ Checklist para os alunos } \\
\hline $\begin{array}{l}\text { 1. Planejar suas atividades } \\
\text { (de resolução do } \\
\text { problema) }\end{array}$ & $\begin{array}{l}\text { Agenda e lembretes das atividades; } \\
\text { Checklist de passos x resolução parcial: } \\
\text { entrada, saída, processamento / } \\
\text { subproblemas, estruturas de programação a } \\
\text { serem utilizadas }\end{array}$ & $\begin{array}{l}\text { Leia com atenção o enunciado do problema; } \\
\text { Pense na saída e entrada do algoritmo e o que precisa } \\
\text { conhecer/calcular para fazer o processamento; } \\
\text { Decomponha o problema em pequenos subproblemas e } \\
\text { resolva um de cada vez; } \\
\text { Lembre das estruturas de programação aprendidas na aula }\end{array}$ \\
\hline $\begin{array}{l}\text { 2. Identificar as } \\
\text { dificuldades }\end{array}$ & $\begin{array}{l}\text { Videoaulas interativas com testes; } \\
\text { Lembretes do conteúdo; } \\
\text { Ferramentas de compilação/depuração; } \\
\text { Ferramentas de testes/ feedback automático; }\end{array}$ & $\begin{array}{l}\text { Fique exercícios simples para identificar em quais estruturas } \\
\text { de programação tem mais dificuldade; } \\
\text { Teste seu programa / faça teste de mesa; }\end{array}$ \\
\hline
\end{tabular}


VII Congresso Brasileiro de Informática na Educação (CBIE 2018)

Anais do XXIX Simpósio Brasileiro de Informática na Educação (SBIE 2018)

\begin{tabular}{|l|l|l|}
\hline & $\begin{array}{l}\text { Material de resumo dos conceitos com erros e } \\
\text { dificuldades mais comuns }\end{array}$ & \\
\hline 3. Conferir as devolutivas & $\begin{array}{l}\text { Nota e feedback das atividades; } \\
\text { Testes/quiz com correção automática }\end{array}$ & $\begin{array}{l}\text { Confira as devolutivas e corrija seus programas; } \\
\text { Confira o gabarito das atividades e provas; } \\
\text { Refaça atividades e avaliações em que não foi bem avaliado }\end{array}$ \\
\hline $\begin{array}{l}\text { 4. Controlar a ansiedade } \\
\text { nas avaliações }\end{array}$ & $\begin{array}{l}\text { Checklist na descrição das atividades sobre a a a a } \\
\text { autorregulação; } \\
\text { Avaliações curtas efeedback frequentes; } \\
\text { Simulados de provas }\end{array}$ & $\begin{array}{l}\text { Não comece diretamente a programar, tome alguns minutos } \\
\text { para planejar sua solução; } \\
\text { Faça rascunhos do algoritmo; } \\
\text { Deixe espaço entre linhas p/ acrescentar código se preciso; } \\
\text { Faça teste de mesa / teste seu programa por partes; }\end{array}$ \\
\hline $\begin{array}{l}\text { 5. Pedir ajuda ao monitor } \\
\text { ou ao professor }\end{array}$ & $\begin{array}{l}\text { Chat, msg/emails, fórum/redes sociais; } \\
\text { Agenda e lembretes (com horários } \\
\text { monitor/professor) }\end{array}$ & $\begin{array}{l}\text { Participe dos plantões de monitoria; } \\
\text { Procure os professores e peça ajuda em seus horários de } \\
\text { atendimento }\end{array}$ \\
\hline $\begin{array}{l}\text { 6. Se reunir com colegas } \\
\text { para estudar }\end{array}$ & $\begin{array}{l}\text { Fóruns, chat, redes sociais, grupos } \\
\text { específicos; } \\
\text { Avaliação por pares; } \\
\text { Encontros/dinâmicas presenciais }\end{array}$ & $\begin{array}{l}\text { Discuta as diferentes soluções para o mesmo problema; } \\
\text { Aprenda com os erros dos seus amigos; } \\
\text { Troque ideias com outras pessoas; } \\
\text { Aumente sua interação social }\end{array}$ \\
\hline
\end{tabular}

\section{Discussões}

Por meio do uso de teorias, trabalhos relacionados e a experiência vivenciada no projeto Met@Aprendiz@gem, este trabalho propôs 11 critérios e checklists que visam auxiliar a inserção e o uso de estratégias de aprendizagem cognitivas e metacognitivas em ambientes virtuais de aprendizagem, além de checklists de recursos e ferramentas para o professor propor aos alunos. Todos os critérios propostos na Seção 4 foram implementados na execução do encontro Desafio PI. Para as estratégias cognitivas, foram desenvolvidas as seguintes ações: 1) Anotar explicações: formulários e papéis fornecidos aos alunos; 2) Fazer resumos, esquemas: fornecidos prontos junto com material de revisão; 3) Elaborar perguntas e respostas: fornecidos exemplos contendo perguntas/respostas junto com material de revisão; 4) Pesquisar materiais extra: fornecidos posteriormente ao encontro; 5) Fixar conceitos e padrões: pontos-chave fornecidos com material de revisão. Como critérios de estratégias metacognitivas, o Desafio PI incluiu: 1) Planejar a resolução: um plano de solução foi inicialmente estabelecido, os passos principais foram destacados e, em seguida, detalhados; 2) Identificar as dificuldades: autoavaliação inicial; depois, em grupo, discutiram dificuldades encontradas, soluções alternativas e identificaram erros; 3) Conferir as devolutivas: gabaritos de todos problemas e vídeo com explicação da solução foram fornecidos posteriormente; 4) Controlar a ansiedade: autoavaliação inicial para autorregulação; correção no mesmo dia e discussão de dúvidas; 5) Pedir ajuda: professores e monitores estiveram disponíveis para dúvidas; 6) Estudar com colegas: o encontro foi presencial e houve a dinâmica em grupos.

Para os critérios cognitivos, muito foi desenvolvido pelos professores e entregue pronto ao aluno. Idealmente, o aluno deve ser estimulado a gerar, por conta própria, suas anotações, resumos, perguntas/respostas, buscar materiais adicionais e identificar/fixar pontos-chave ou padrões de problema-solução. A partir dos exemplos entregues, o aluno pode passar a considerar essas boas práticas se, por exemplo, o professor passar atividades que o faça desenvolver materiais similares e a usá-los como fonte de consulta durante provas. Analogamente para os critérios metacognitivos, o que foi realizado durante o encontro deve ser incentivado para que os alunos adotem as estratégias com frequência ao longo de seu percurso na disciplina, por exemplo, 
VII Congresso Brasileiro de Informática na Educação (CBIE 2018)

Anais do XXIX Simpósio Brasileiro de Informática na Educação (SBIE 2018)

incluindo critérios de avaliação que valorizam tais estratégias ou realizando atividades que as favoreçam.

$\mathrm{O}$ índice de reprovação entre as turmas de PI-semipresencial nos últimos três quadrimestres ideais, 2016.Q1, 2017.Q1 e 2018.Q1, caiu consideravelmente, com valores respectivos de $38,1 \%, 28,1 \%$ e $22,2 \%$, envolvendo turmas que finalizaram o período letivo com 147, 185 e 162 alunos respectivamente. As condições das aulas e os critérios de avaliação nos três quadrimestres foram essencialmente similares, de modo que é possível comparar tais índices de reprovação com boa margem de confiança. Os materiais instrucionais, conjunto de atividades, e estilo e quantidade de provas foram idênticos. Os enunciados das questões das provas sempre foram diferentes para cada turma, porém com mesmo nível de dificuldade e critérios de correção equivalentes. Em 2016.Q1, o projeto não havia sido iniciado; em 2017.Q1, ocorreram as primeiras ações do projeto com a revisão e produção de novos materiais; em 2018.Q1 foi realizado o encontro Desafio PI junto com outras iniciativas como: videoaulas interativas e vídeos dicas (Você Sabia?), cujos resultados ainda são incipientes.

Desse modo, aparenta ser promissora a adoção dos critérios definidos. Entretanto, reunir todos eles em um ambiente virtual de aprendizagem não é trivial, pois estes, em geral, não estão nativamente adequados para que o professor insira e trabalhe com as estratégias. Em particular, o Desafio PI foi realizado presencialmente (apesar da turma ser semipresencial e as aulas serem oferecidas em formato $\mathrm{EaD}$ ), para superar a limitação das ferramentas de colaboração e comunicação dentro do AVA.

\section{Considerações Finais}

Várias pesquisas indicam que mobilizar estratégias cognitivas e metacognitivas adequadas à determinada situação de aprendizagem pode melhorar consideravelmente $o$ desempenho dos alunos. Entretanto, há poucos recursos e ferramentas disponíveis em ambientes computacionais que favorecem o seu uso por alunos, principalmente em atividades anteriormente preparadas pelo professor. Nesse contexto, este artigo descreveu 11 critérios que apoiam o uso das estratégias cognitivas e metacognitivas pelo aluno no desenvolvimento de lógica de programação, e apresenta checklists que relacionam essas estratégias de aprendizagem com recursos e ferramentas que podem auxiliar o professor a promover o uso dessas estratégias em ambientes computacionais. Os critérios selecionados priorizam as estratégias identificadas como as menos adotadas por alunos de disciplina introdutória de programação em ambiente de ensino a distância.

Como trabalhos futuros, é necessário definir mecanismos para implementar essas estratégias em ambientes virtuais de aprendizagem, bem como fazer uma validação da eficácia dessas estratégias em disciplinas semipresenciais.

\section{Referências}

Alaoutinen, S., Smolander, K. (2010). "Student self-assessment in a programming course using bloom's revised taxonomy”. In Proc. 15th ITiCSE, 155-159.

Alhazbi, S., Hassan, M. (2010). "Fostering Self-Regulated learning in Introductory Computer Programming Course”. APEC'2008 Education Reform Symposium in Xi'na, China. (2008). 21st Century Competencies.

Bergin, S., Reilly, R., Traynor, D. (2005). "Examining the role of self-regulated learning on introductory programming performance". In Proc. 1st ICER, 81-86. 
VII Congresso Brasileiro de Informática na Educação (CBIE 2018)

Anais do XXIX Simpósio Brasileiro de Informática na Educação (SBIE 2018)

Beyer, B.K. (1985). Critical thinking: What is it? Social Education, v. 49, n.4 p. 270-76.

Boruchovitch, E. (1999). Estratégias de aprendizagem e desempenho escolar: considerações para a prática educacional. Psicologia: Reflexão e Crítica,12, 361-376.

Brown, A.L. (1987). Metacognition, executive control, self-regulation, and other more mysterious mechanisms. In F. E. Weinert \& R. Kluwe (Orgs.), Metacognition, motivation, and understanding (pp. 1-16). Hillsdale, N. J.: Erlbaum

Brusilovsky P., Sosnovsky S.(2005). Individualized exercises for self-assessment of programming knowledge: an evaluation of QuizPACK .J.Educ. Resour. Comput.5(3)

Castro, R.F. (2016). Autorregulação da aprendizagem no ensino superior a distância: o que dizem os estudantes?. Revista Brasileira de Ensino Superior, v. 2, n. 2, p. 15-26.

Chen, C.S. (2002). Self-regulated learning strategies and achievement in an introduction to information systems course. Information Technology, Learning and Performance Journal, v. 20, n. 1, p. 11-25.

Fantinel, P. C., Angelo, N., Angelo, D., Maraschin, C. (2013). Autorregulação da aprendizagem na educação a distância online. In: XVIII Conferência Internacional sobre Informática na Educação.

Flavell, J. H. (1976). Metacognitive aspects of problem solving. In L. B. Resnick (Ed.), The nature of intelligence, 231-235.

França R., Tedesco P. (2014). Um modelo colaborativo para a aprendizagem do pensamento computacional aliado à autorregulação, XXV SBIE.

Francisco R., Pereira Jr C., Ambrósio A. P. (2016) Juiz Online no ensino de Programação Introdutória - Uma Revisão Sistemática da Literatura. XXVII SBIE

Goya, D.H.; Rodriguez, C.L.; Araujo, C.A.; Venero, M.L.F. (2017). O Uso de Estratégias de Aprendizagem Cognitivas e Metacognitivas na Disciplina Semipresencial de Processamento da Informação. In: III Workshop de Ensino em Pensamento Computacional, Algoritmos e Programação (WAlgProg).

Havenga M., Breed B., Mentz E. (2013). Metacognitive and problem-solving skills to promote self-directed learning in computer programming: teachers'experiences, SAeDUC, v. 10, n. 2.

Havenga M. (2015). The role of metacognitive skills in solving object-oriented programming problems: a case study, TD: The Journal for Transdisciplinary Research in Southern Africa, v. 11, n. 1, 133-147, AOSIS.

Kautzmann T. R., P. Jaques P. (2016) Treinamento da Habilidade Metacognitiva de Monitoramento do Conhecimento em Sistemas Tutores. Revista Brasileira de Informática na Educação, v. 24, n. 2

Koutselini, M. (1991) Child development and school reality. Nicosia: Pedagogical Institute of Cyprus.

Leite E., Darsie M. (2011). Implicações da metacognição no processo de aprendizagem da Matemática, Revista Eletrônica da Educação, v. 5, n.2.

Lishinski A., Yadav A., Good J., Enbody. R. (2016). Learning to Program: Gender Differences and Interactive Effects of Students' Motivation, Goals, and Self-Efficacy 
VII Congresso Brasileiro de Informática na Educação (CBIE 2018)

Anais do XXIX Simpósio Brasileiro de Informática na Educação (SBIE 2018)

on Performance. In Proc. of the 2016 ACM Conference on International Computing Education Research (ICER '16). ACM, NY, USA, 211-220.

Moreira, A.E.C. (2014). Relações entre as estratégias de ensino do professor, com as estratégias de aprendizagem e a motivação para aprender de alunos do Ensino Fundamental 1. Dissertação. Universidade Estadual de Londrina

Moreira M. P., Favero, E. L. (2009). Um Ambiente Para Ensino de Programação com Feedback Automático de Exercícios. In: Workshop Sobre Educação e Computação Anais do XVIII Congresso da Sociedade Brasileira de Computação 2008.

Ngai, G., Lau, W. W., Chan, S. C., Leong, H. V. (2009). On the implementation of selfassessment in an introductory programming course. ACM SIGCSE Bulletin, 41(4), 85-89.

Parham, J., Gugerty, L., Stevenson, D.E. (2010). Empirical evidence for the existence and uses of metacognition in computer science problem solving. In Proc. 41st SIGCSE, 416-420.

Pimentel E. P. P, Omar N., França V. F. (2005) Um Modelo para Incorporação de Automonitoramento da Aprendizagem em STI. Revista Brasileira de Informática na Educação, v. 13, n. 1.

Rodriguez, C.L.; Goya, D.H.; Venero, M.L.F; Araujo, C.A. (2017) Met@ Aprendiz@gem: estratégias metacognitivas e recursos diferenciados para apoiar a construção do pensamento lógico, XXVIII SBIE.

Santos, A.A. A., and Boruchovitch, E. (2008). Escala de estratégias de aprendizagem para Universitários, Manuscrito não publicado, Unicamp, Campinas, Brasil.

Silva, A. L.; Sá, I. (1997). Saber estudar e estudar para saber. Porto Editora.

Sirotheau, S.; de Brito, S. R.; da Silva, A. D. S.; Eliasquevici, M. K.; Favero, E. L.; Tavares, O.D.L. (2011). Aprendizagem de iniciantes em algoritmos e programação: foco nas competências de autoavaliação. XXII SBIE.

Sitthiworachart, J., Joy, M. (2004). Effective peer assessment for learning computer programming. In Proc. 9th SIGCSE, 122-126.

Son, L. K.; Schwartz, B. (2002) The relation between metacognitive monitoring and control. In: Perfect, T. J.; Schwartz, B. Applied Metacognition. Cambridge: UK Cambridge University Press.

Testa, M. G.; Freitas, H. A. (2006). Importância da autorregulação dos recursos da aprendizagem para a efetividade dos cursos desenvolvidos na internet. In: XXX Encontro da ANPAD (ENANPAD), 30.

Warr, P.; Allan, C. (1998). Learning strategies and occupational training. International Review of Industrial and Organizational Psychology, 13, 83-121.

Weinert, F. E.; Kluwe, R. H. (1987). Metacognition, motivation, and understanding. Hillsdale, N. J.: Erlbaum.

Weinstein, C.E.; Mayer, R.E. (1985). The teaching of learning strategies. Em M. Wittrock (Org.), Handbook of research on teaching (p. 315-327). NY, Macmillan. 